\title{
Experiences of women regarding gaps in preconception care services in the Iranian reproductive health care system: A qualitative study
}

\author{
Roghieh Bayrami ${ }^{1}$, Robab Latifnejad Roudsari ${ }^{2}$, Hamid Allahverdipour ${ }^{3}$, Mojgan Javadnoori ${ }^{4}$, Habibollah \\ Esmaily
}

${ }^{1}$ Ph.D. Student in Reproductive Health, Department of Midwifery, School of Nursing and Midwifery, Student Research Committee, Mashhad University of Medical Sciences, Mashhad, Iran

${ }^{2}$ Research Centre for Patient Safety, Department of Midwifery, School of Nursing and Midwifery, Mashhad University of Medical Sciences, Mashhad, Iran

${ }^{3}$ Department of Health Education and Promotion, Tabriz University of Medical Sciences, Tabriz, Iran

${ }^{4}$ Reproductive Health Promotion Research Center, Faculty of Nursing and Midwifery, Ahvaz Jundishapur University of Medical Sciences, Ahvaz, Iran

${ }^{5}$ Health Sciences Research Center, Department of Epidemiology and Biostatistics, School of Health, Mashhad University of Medical Sciences, Mashhad, Iran

\section{Type of article: Original}

\begin{abstract}
Introduction: Despite the beginnings of preconception care (PCC) delivery around a decade ago in Iran, there are still significant gaps in its service delivery. The purpose of this study was to explore the perceptions and experiences of women as well as midwives toward gaps in PCC delivery in the Iranian reproductive health care system.

Methods: In this exploratory qualitative study, 27 married women and 13 midwives were recruited using purposive sampling from five health centers in Mashhad, Northeast of Iran. Respondents participated in semistructured, in-depth, individual and focus-group interviews to express their perceptions and experiences about gaps in PCC. Data were analyzed using conventional content analysis adopted by of Graneheim and Lundman (2004) with MAXQDA software.

Results: Analysis of data revealed four themes: 1) missing men and adolescents from PCC; 2) insufficient PCC package; 3) inadequate PCC strategies; and 4) health care providers' incompetency.

Conclusion: It is recommended to deliver gender-sensitive PCC through addressing couples' instead of just women's PCC and to take into account the adolescent girls' health in order to improve their preconception health. Standardization of protocols and attention of health professionals toward occupational-environmental hazards and sexual and reproductive issues as well as enhancing professional capability of health care providers could improve PCC service delivery.

Keywords: Preconception care, Reproductive Health Care, Women, Qualitative Study, Iran
\end{abstract}

\section{Introduction}

Preconception care (PCC) has always been one of the major segments of health care and health promotion strategies for women in reproductive age, the main aim of which is health promotion, along with screening and intervention for women in reproductive age in order to reduce risk factors that possibly affect later pregnancies. In other words, PCC is part of a wide care model that results in healthier women, children and families in the future $(1,2)$. According to reports of the World Health Organization (WHO), PCC lies in the setting of sexual and reproductive health (3). There is strong evidence that suggests those promoting women's health before pregnancy has favorable

\section{Corresponding author:}

Robab Latifnejad Roudsari, Ebne-Sina Street, School of Nursing and Midwifery, Mashhad University of Medical Sciences, Mashhad, Iran.

Tel: +98.9370400607, Fax:+98.5138597313, E-mail: latifnejadr@mums.ac.ir and rlatifnejad@yahoo.com Received: December 13, 2015, Accepted: February 06, 2016, Published: November 2016 iThenticate screening: January 24, 2016, English editing: August 02, 2016, Quality control: October 06, 2016 (C) 2016 The Authors. This is an open access article under the terms of the Creative Commons Attribution-NonCommercialNoDerivs License, which permits use and distribution in any medium, provided the original work is properly cited, the use is non-commercial and no modifications or adaptations are made. 
fetal and maternal outcomes and reduces pregnancy complications, birth defects, fetal death, low birth weight, and pre-term labor (1). On the other hand, according to the report by the Center of Maternal and Child Health Research, PCC is an effective factor in reducing maternal mortality rate $(4,5)$. Although it has been over 20 years for which the concept of preconception health and care has been considered in developed countries, and started about a decade ago in Iran, in many countries, many health problems, including unwanted pregnancy, unsafe abortion, genetic diseases, and preterm labor, have remained unsolved (6) in such a way that, according to the report by the WHO, $5 \%-7 \%$ of live births in developed countries are preterm labor. It is estimated that this rate is higher in developing countries (7). In the United States, nearly half of pregnancies are unwanted (8). In a meta-analysis study conducted by Moosazadeh et al., prevalence of unwanted pregnancy in Iran is $31.6 \%$ (9). According to results of different studies, prevalence of unwanted pregnancies in Mashhad has been reported to be $35.8 \%-37 \%(10,11)$. On the other hand, it should be considered that, in order to develop, implement, and maintain preconception health policies, data are required to be used in line with developing health care standards in health services, while such data are not available in most countries of the world (12), including in Iran. In addition, because the world strategy of pregnancy health should be converted into approaches of each country, implementing plans related to that is the independent right of any nation that should be compatible with national regulation and development priorities of that nation and to be implemented with full respect toward religious, cultural, and moral values of that nation while in harmony with universal human rights (13). Obviously, the structure of a PCC plan is different in international communities based on the type of health system and economic level. However, most countries are willing to have the best organized health system. Therefore, extensive policies and instructions are used to regulate PCC services in a health system (14). Therefore, considering the existing gaps in the lack of PCC standards, this study was designed with the aim of investigating existing gaps in PCC. Because most experiences, perceptions, and beliefs that establish human health and care needs cannot be measured using quantitative research approaches, it is required to evaluate and assess these cases in a proper and capable way, including qualitative research methods. Qualitative approaches provide a rich and deep description of human phenomena and experiences $(15,16)$; therefore, they are regarded as a proper method to study this issue. Considering the lack of qualitative studies in line with deep understanding of deficits and gaps related to PCC, this qualitative study was conducted to explore perceptions and experiences of woman in reproductive age in regards to existing gaps in PCC services.

\section{Material and Methods}

\subsection{Research design and setting}

This study, which is a qualitative content analysis, is part of an extensive mixed methods study, which is conducted to develop a descriptive model for prediction of preconception health behavior of Iranian women in Mashhad in 2014. Qualitative content analysis can be used in cases where the aim of the study is to obtain an extensive and comprehensive description of a phenomenon, thus creating a holistic and new approach and finally creating themes or categories that explain the phenomenon under study (17). In this study, to reflect women's and midwives' experiences and feelings about existing gaps in PCC of the health system, qualitative content analysis was used. The study population was women seeking pregnancy covered by 30 medical and health centers selected from five health centers of Mashhad. In addition, to complete the data, midwives providing PCC services in the health and medical centers are included in the study.

\subsection{Inclusion and exclusion criteria}

Inclusion criteria were being married, Iranian, and willing to participate in the study. Women were between 15 and 49 years of age and lived with their husband in the same house when they took part in the study. Infertile women were excluded from the study because PCC in these women is different from that in fertile women, i.e., it was more specialized basically. Inclusion criteria for midwives, as preconception service providers, were having at least three years of work experiences in health and medical centers.

\subsection{Sampling}

In this study, a purposive sampling method with a strategy of maximum variation was used. To select participants, at first the author referred to urban health and medical centers covered by five health centers of Mashhad and then, by coordination with related authorities and explaining the aim of the study, women who referred to these centers and were willing to share their experiences and had enough time were invited to participate in the study. Participants were selected by maximum diversity in terms of age, number of children, pregnancy rank, educational level, employment status, having or lacking PCC, economic and social status, and even the center that covers them. Maximum diversity in service providers (midwives) was considered based on factors such as work experience and 
location of health centers. Finally, 27 married women in reproductive age and 13 midwives were selected as participants of this study.

\subsection{Interviews and data collection}

In this study, to collect qualitative data, semi-structured, in-depth individual and focus group interviews were used. For cultural sensitivity of the issue under study, it was predicted that people may not be able to present their experiences or views in public. Therefore, individual face-to-face semi-structured interviews were conducted. At the beginning of each interview, the researcher introduced herself and explained the aims of the study to the participants and then obtained written informed consent to conduct the interview. The author tried to provide the background for a better interview by asking a series of preliminary questions and creating a good relationship through attracting their trust and providing a relaxed environment for the participants. Interviews with open-ended questions allowed participants to explain their experiences on intended phenomena completely (18). The interview started by asking a general question such as "would you please tell me about your PCC experiences?" and continued by asking other open questions on the way of how to conduct PCC, along with its demands and shortcomings. For more clarity, other probing questions were asked such as requesting more details or giving examples. In this study, after conducting an empirical pilot interview with a woman who met inclusion criteria and receiving her feedback, some changes were made on questions. Nonverbal behaviors of participants, including their facial expressions, tone of voice, and their emotional reactions along with important notes of each meeting were recorded immediately after the interview. In cases that it was not possible to continue the interview because of unexpected events, it was stopped and postponed to the next session if it was accepted by the participant. Depending on the situation, each interview in the service providers' group and woman's group lasted for $90 \mathrm{~min}$ and 45-100 minutes, respectively. In this regard, a supplementary interview was conducted with one of the participants. In this study, two focus groups were formed with women separately. Women's meetings were held in one of the health centers with previous coordination and invitation. At the beginning, the researcher gave some general details to inform all members about the quality of group discussion. The interview started with a general question and continued with more specialized questions in line with objectives of the study. Each focus group interview consisted of five members. Participants sat in a circle at a table. Discussions were recorded using two MP3 players on both sides of the table, and personal characteristics of participants were recorded. A female colleague, who was the facilitator of the meeting, took notes from marginal events and managed time. Group discussions lasted for 60 to 90 minutes. All interviews were recorded using an MP3 player. Each interview was immediately transcribed verbatim before conducting the next interview, and data analysis was conducted using qualitative content analysis and MAXqda software. The transcripts were read several times to immerse the author in the data to achieve a general view toward the data. Then, following qualitative content analysis principles of Graneheim and Lundman, each interview was regarded as a unit of analysis as a whole, and it was specified as meaningful units with meaningful sentences and words related to one meaning. Using the data reduction rule, condense meaningful units were obtained. Then, codes were extracted using an abstraction process, and proper titles or labels were attributed to them. Samples of data reduction included code creation, categories, and themes. Primary codes were formed in this way, and, following that, abstract codes were extracted considering presumption and author's perception of similarities of primary codes (19).

\subsection{Rigour of findings}

In this study, to assure the rigour of the findings, based on suggested measures of Lincoln and Guba, issues of credibility, dependability, confirmability, and transferability were used (20). Credibility of the study was verified using various methods, including prolonged engagement with participants with maximum variation, review of findings by participants of the study and confirming them by the project supervisor. To this purpose, some sample of coded data was taken to the participants to ask for the quality and accuracy of researchers' impression. In addition, some samples of coded data and interpretations were reviewed by other researchers experienced in conducting qualitative studies, and validity of the interpretations was confirmed. For dependability, a decision trail of the research process was provided, which made other researchers capable to audit the research process. Confirmability of findings was evaluated through checking the process of study by the external reviewers familiar with qualitative research. To maintain transferability, main findings of the study were presented to some women who did not take part in the study but had a similar profile for participants in this study, which was confirmed.

\subsection{Research ethics}

Before being conducted, this study was approved by Regional Committee of Ethics in Research in Mashhad University of Medical Sciences, and informed consent was obtained from all participants. 


\section{Results}

The population under study consisted of women covered by health centers in five districts as well as midwives employed in health centers of Mashhad. Participants were between 18 and 45 years of age, most of whom had a high school degree to diploma and were a housewife. In addition, midwives, both graduate and post-graduate, were considered as key informants in PCC. First, 878 primary codes were extracted from content analysis of 40 interviews and after examining the codes and identifying similarities as well as reducing them based on overlap rule; finally, 220 main codes were extracted; in later stages, they manifested in four main themes and nine categories (Table 1). Each categories is explained in detail along with cited quotations of participants in the following.

Table 1. Main themes and categories emerged through data analysis

\begin{tabular}{|c|c|c|}
\hline Main theme & Categories & Subcategories \\
\hline \multirow[t]{6}{*}{$\begin{array}{l}\text { Missing men and } \\
\text { adolescents from PCC }\end{array}$} & \multirow[t]{2}{*}{ Men's absence in PCC } & $\begin{array}{l}\text { Lack of men's participation in the planning for } \\
\text { pregnancy }\end{array}$ \\
\hline & & Failure to provide PCC for men \\
\hline & \multirow[t]{2}{*}{$\begin{array}{l}\text { Neglecting adolescent girls in terms } \\
\text { of preconception health }\end{array}$} & $\begin{array}{l}\text { Not considering adolescent girls' training for } \\
\text { preconception health }\end{array}$ \\
\hline & & $\begin{array}{l}\text { Shortcoming in providing care to girls before } \\
\text { marriage }\end{array}$ \\
\hline & \multirow[t]{2}{*}{ Low public awareness of PCC } & $\begin{array}{l}\text { Insufficient low awareness of preconception } \\
\text { health behaviors among men }\end{array}$ \\
\hline & & $\begin{array}{l}\text { Inadequate awareness of preconception health } \\
\text { behaviors among women }\end{array}$ \\
\hline \multirow[t]{4}{*}{ Insufficient PCC package } & \multirow[t]{2}{*}{ Poor protocol for counseling items } & $\begin{array}{l}\text { Deficient protocol for birth control methods } \\
\text { counseling }\end{array}$ \\
\hline & & $\begin{array}{l}\text { Failure to include domestic violence } \\
\text { counseling in protocol }\end{array}$ \\
\hline & \multirow[t]{2}{*}{$\begin{array}{l}\text { Neglecting sexual and reproductive } \\
\text { issues }\end{array}$} & $\begin{array}{l}\text { Not educating clients regarding reproductive } \\
\text { cycles }\end{array}$ \\
\hline & & Poor sexual health education \\
\hline \multirow{4}{*}{$\begin{array}{l}\text { Inadequate PCC } \\
\text { strategies }\end{array}$} & \multirow{2}{*}{$\begin{array}{l}\text { Lack of integrating PCC into other } \\
\text { health care services }\end{array}$} & Not integrating PCC in child care program \\
\hline & & $\begin{array}{l}\text { Lack of considering PCC in women's health } \\
\text { care referrals }\end{array}$ \\
\hline & \multirow{2}{*}{$\begin{array}{l}\text { Lack of attention to socio- } \\
\text { environmental determinants of } \\
\text { health }\end{array}$} & Not being covered by insurance \\
\hline & & $\begin{array}{l}\text { Not considering socio-environmental factors in } \\
\text { PCC }\end{array}$ \\
\hline \multirow{4}{*}{$\begin{array}{l}\text { Health care providers' } \\
\text { incompetency }\end{array}$} & \multirow[t]{2}{*}{ Poor caregivers' performance } & Poor caregivers' relationship with their clients \\
\hline & & $\begin{array}{l}\text { Inadequate information and education of } \\
\text { clients by caregivers }\end{array}$ \\
\hline & \multirow[t]{2}{*}{ Inadequate staff counseling skills } & $\begin{array}{l}\text { Inadequate skills of caregivers regarding } \\
\text { preconception birth control methods } \\
\text { counseling }\end{array}$ \\
\hline & & Inadequate skills in couple counseling \\
\hline
\end{tabular}

\subsection{Men's Absence in PCC}

Analysis of interviews revealed that most participants of this study believed that, in our society, men do not have enough participation in planning for pregnancy. One participant in this study stated: "Unfortunately, in our society, men have plans for their study, work, vacations, entertainment, or even their retirement. But they do not plan for pregnancy and healthy fetus" (27-year-old mother, employed, interconception care). Most participants believed that having a baby is a mutual decision between couples, and they are both responsible for pregnancy health and future children. Therefore, they should do their best to obtain favorable results, i.e., safe pregnancy and childbirth and healthy children. A mother said, "Men are responsible for their children's health, too. They should be sensitive on their own and their children's health. They also should be careful. They should be sure about their health. At least, it should be in a way that they attend training courses, too" (30-year-old mother, housewife, associate diploma, interconception care). In addition, most participants of the study have confessed that men's health is not considered in care system of the country. Analysis of interviews showed that both women and midwives who provide PCC 
believe in the necessity of PCC for men. "Only women are considered in PCC plans, and men's health is neglected or ignored" (postgraduate midwife, 12 years of work experience). One of the midwives attending this study stated: "It should be considered that the father's health affects the child's health, too. It is obvious that the father's genetics, risky behaviors of the father, such as drug or alcohol abuse, and sexually transmitted diseases affect fetal health. We do not pay attention to these facts, and we believe that only mothers should be cared for" (Postgraduate midwife, eight years of work experience).

\subsection{Neglecting adolescent girls in terms of preconception health}

Most participants of the study believed that adolescent girls' health is of special importance. Adolescent-hood of girls is the infrastructure and beginning that directs later stages of their lives and has a direct effect on their family and children's health. One of the midwives participated this study stated: "Today's girls are tomorrow's mothers and the health key of current and later generations. Their health is important. Mostly, this age group does not have required information on pregnancy and health. They may get married at this age and soon become pregnant, without having required knowledge ... I believe that PCC should be started at adolescence" (graduate midwife, 10 years of work experience). Most participants have pointed out the necessity to start PCC before marriage and even before high school. They believed that adolescent girls' health is less considered, and they do not have required knowledge on pregnancy health. One of the participants of the study stated that: "I believe there are some things we should know before marriage. It is better that schools give us this information. After marriage, couples do not know each other well, and health issues are less considered. Singlehood is the best opportunity for us to check our health. However, it is not considered at all" (33-year-old mother, housewife, interconception care).

\subsection{Low Public Awareness of PCC}

Findings of the study demonstrated that low awareness on preconception health behavior was not only among husbands and relatives of women who participated in this study, but also it was obvious in women who had PCC. This was obvious from the answers they gave to the question related to PCC. On the other hand, women who had PCC were not informed well on intended behavior. They just believed in usefulness and uselessness of a behavior, and they did not have enough information on the cause of its usefulness. One of the participants said: "I just know that I should take folic acid three months before getting pregnant, but I don't know why and what its effect is" (18year-old mother, housewife, diploma, PCC). Another participant said: "Nobody knows anything about PCC in my family. My mother-in-low made fun of me when she realized I referred for preconception checkup. My sister-in-law, who is a graduate, told me 'Don't take your child to the doctor already, for God's sake!' What are you doing?" (31year-old mother, middle school, housewife, interconception care). Other findings of the study revealed that not only do people in the society require to be informed because of unawareness, but their attitude toward preconception behaviors is unfavorable. One of the midwives participated this study stated: "Our problem is that our target group has not yet believed that what the benefits of PCC are. They do not believe in PCC similar to pregnancy care" (graduate midwife, eight years of work experience).

\subsection{Poor Protocol for Counseling Items}

Most midwives participating in this study considered the lack of performance protocol for counseling items as the gaps available in PCC in the health system of the country. One of the midwives participating in this study stated: "There is no specified instruction through which we are able to say how and when to stop birth control to become pregnant. There is no specified protocol using which we know when to stop birth control for pregnancy. Therefore, these should be specified and they should give us advices using that protocol" (postgraduate midwife, eight years of work experience). Most midwives believed in having a specified protocol for counseling. For example, one of the midwives considering this issue stated that, "There is no specified guideline for women under violence who are in preconception period based on which we are able to care them" (graduate midwife, 10 years of work experience).

\subsection{Neglecting Sexual and Reproductive Issues}

Interview analysis showed that women do not usually have sufficient information and awareness about the anatomy of the reproductive system and reproductive system physiology, and one of the main problems of PCC is that birth control methods are introduced to them immediately and without being prepared; this will confuse them and result in their lack of attention to these issues. A woman in this study stated: "We are not aware of women's reproductive system because we are not that much educated. We do not know how pregnancy occurs and how pills and IUD work. If we know this, we can regulate our pregnancy better" (29-year-old mother, high school, interconception care). Although the role of sexual relations in physical, mental, and social health is confirmed, it is not considered in practice in health programs. On the other hand, health staff refrain from talking about sexual issues, and women are 
not willing to ask about sexual issues because of cultural restrictions, shyness, etc. "It will be better to teach us in a health course how we can become pregnant within a desired period. I stopped taking folic acid. Unfortunately, they do not tell us many issues in the health course; as if they are shy of talking about these issues as well" (27-year-old mother, high school, housewife, first PCC). Request for determining fetal gender is among women's expectations when becoming pregnant. When hearing about PCC, most women think that determining fetal gender is part of this care, too. One of the participants said, "First, I thought that PCC is for determining fetal gender. Then I realized that it is something different" (22-year-old mother, diploma, housewife, first PCC).

\subsection{Lack of Integrating PCC into Other Health Care Services}

Most midwives participating in this study pointed out the integration of PCC with other health care. For instance, one of the midwives said: "Suppose that a mother with epilepsy refers a neurologist to control her disease. The neurologist should ask her about her pregnancy plans and recommend her to take, for example, twice as much folic acid supplements if required. Maybe that woman would never refer to a health center. There should be a guideline to be communicated to physicians to take this issue as serious" (42-year-old midwife, 16 years of work experience). Most women in this study stated: "These cares should be repeated periodically and have reminders. For example, it would be better to talk about these cares in before marriage classes" (32-year-old mother, B.A., employed, interconception care). Data analysis suggested midwives believed that any opportunity should be used for PCC. In such a way that one of the midwives stated: "It should be noted that PCC is not just for a midwife and gynecologist. It is rare that a woman asks for care. Therefore, all these specialties should take advantage of any opportunity to promote preconception behavior."

\subsection{Lack of Attention to Socio-Environmental Determinants of Health}

Most participants stated there is not sufficient awareness and information on environmental and occupational risk factors given to clients. A participant said: "When a woman decides for pregnancy, she should have complete information on risk factors affecting fetus ... Of course, I study a lot, and I know these issues. For example, I know that the cosmetics we use these days are full of lead; air population is harmful to a fetus as well as smoking and washing detergents. However, they never tell us about these-neither in health centers nor in offices. I have studied and I know these. What about others? They should tell us. Unfortunately, these issues are not pointed out at all" (25year-old mother, B.A., housewife, first PCC). Some participants believed that economic and social factors of clients should be considered in all care programs, including PCC and counseling. One of the participants said: "In health centers, they say, have fish, fruits, milk! ... Everybody can tell us these. But the art is that they tell poor and lowincome people how to have a balanced diet according to their income level" (27-year-old mother, diploma, housewife, PCC).

\subsection{Poor caregivers' performance}

Not allocating sufficient time for clients by health providers is one of the factors affecting unwillingness of most mothers to conduct health behaviors. "Can they (health providers) really do these? I mean, do they have time? They are pretty busy. I think they just know how to fill out a form to finish work, and then they have no responsibility. They just know how to fill out a form correctly or incorrectly to disclaim responsibility. Instead of spending time on training people, they fill out forms. They just want to put people off' (32-year-old mother, M.A., employed, first PCC). Contradictory results were obtained from data analysis on women's perception relative to effectiveness of services provided by service providers, in such a way that few participants acknowledged effectiveness of midwives. "I referred to a gynecologist in addition to a health center. Ms. X in the health center told me all issues that the gynecologist has told me in her office. Even it seems that Ms. X knew her job better that gynecologist" (28-year-old mother, diploma, interconception care). On the contrary, most women were not fully satisfied by services provided by health staff. "That's right, in the health center, they told us to take folic acid supplements and to take a certain test. But they do not give us information as we want. They do not take it seriously, and they just want to put a client, who has referred them, off' (25-year-old mother, housewife, first PCC). Most women have mentioned bad tempers and inappropriate behavior of service providers... "There is something on my mind that they should be a little nicer. I'm afraid of referring health centers, and my husband says this as if I want to go to the war. I know they are very busy and become tired. I always refer them early in the morning, and I try to be there at 8 a.m. with fear. Their behavior prevents us from asking questions" (34-year-old mother, B.A., employed, interconception care).

\subsection{Inadequate staff Counseling Skills}

Most midwives participating in this study regarded counseling as part of PCC that includes various fields, including pregnancy prevention methods, nutrition, physical exercises, and medical diseases. They believed that a counselor 
should have required technical and communicative skills for counseling. "It could be said that preconception counseling is in fact identifying and preventing medical complications. In addition, various mental-social complications occur during pregnancy, the bad consequences of which affect the mother and infant. That's right: the midwife conducts the counseling and care. They have part of the information and knowledge related to this field. But I confess, between me and you, who has told us about counseling methods? Counseling requires special skills. It requires taking courses, and none of us have taken such a course. Therefore, our efficiency diminishes. Clients are not similar to prescribe the same thing for all of them" (28-year-old midwife, three years of work experience). Another midwife stated: "Every group should be counseled separately, for example, counseling couples, adolescents and males. However, we do not have such skills" (32-years-old midwife, eight years of work experience).

\section{Discussion}

This study was conducted for the first time in Iran to qualitatively explore gaps in the PCC services. Four themes were extracted from interviews' analysis associated with gaps available in PCC that include "missing men and adolescents from PCC," "insufficient PCC package," "inadequate PCC strategies" and "health care providers' incompetency." All concepts available in the data were associated with gaps in reproductive health care system. Findings extracted from interviews confirmed that most participants of the study had acknowledged men's absence in PCC. PCC for men focuses on preventive strategies used in preconception to improve pregnancy and fetal outcomes (21). Recently, the Center for Disease Control and Prevention has published PCC for men (22). Results from the study conducted by Choiriyyah have revealed that about $60 \%$ of $15-44$-year-old men require PCC, and the highest necessity was observed among men with 15-29 years of age. Obviously, the need for PCC was different according to background characteristics of men (23). On the other hand, public access to reproductive health until 2015 has been part of the new millennium development goals (MDGs); some criteria of which are the amount of birth control method used by adolescents. To improve these criteria along with presenting birth control services, it is required to add PCC to health care to improve women and girls' health and nutrition (3). As previously mentioned, although PCC has been started for about a decade, it is not yet known as routine care similar to pregnancy care, and it is not yet welcomed. In the present qualitative study, women who have not had PCC or have not conducted fine behaviors correctly and completely, they mentioned unawareness of its usefulness as the cause of lack of care or fine behaviors. Findings of a qualitative study conducted by Tuomainen et al. comply with findings of this study (24). In a study by Bayrami et al., awareness and performance of health care staff was not been favorable in terms of PCC (25); in a study conducted by Riazi, about $80 \%$ of pregnancies had been programmed, about less than $10 \%$ of women had taken folic acid supplement, and taking folic acid was not related to the history of delivering infants with a neural tube defect (26).

Poor protocol for counseling items was another defect in PCC. In terms of PCC in Iran, there are comprehensive but general guidelines; however, no performance protocol is defined for counseling items, including domestic violence and using birth control methods. Developing executive protocol is one of the requirements of evaluating quality of care and as a warranty for feasibility of care delivery. Results of the present study comply with results of the study conducted by Merghati Khuiee et al. on this issue (27). By having the view of "determining fetal gender by PCC," most participants believed that they can plan for favorable fetal gender determination by PCC. As they believed, couples should not be worried and stressed for determining their future child's gender, which was important for most women. Although we have frequently heard that "boys and girls are the same and a healthy child is more important," the reality is that peoples' hearts think differently. In fact, a human is a selective creature, and had long dreamed of determining his or her child's gender. Although gender determining services are not provided in state health centers, all women refer to private offices with the view to determine the gender of their fetus. They believe that, by selecting fetal gender, they regulate family dimensions. In addition, as they believe, the desire to have, for example, a daughter in the family results in second, third, and ... pregnancies with the hope of having a girl. Obviously, preventing the birth of infants with gender-related genetic diseases such as hemophilia is the main objective of PCC that should be considered. On the other hand, emphasizing health behaviors before pregnancy such as taking folic acid supplements and conducting screening three months before pregnancy have created this expectation in clients that they should become pregnant after three months of starting these behaviors; otherwise, they will be disillusioned and put aside health behaviors such as observing nutrition, environmental risk factors, and taking folic acid supplements. Therefore, it seems that giving required awareness on reproductive and sexual issues by talking about reproductive physiology and menstrual cycle in preconception counseling sessions should be provided for clients to show proper performance both for birth control and planning for pregnancy. Results from analysis of interviews showed that according to midwives, any opportunity should be employed for PCC. In this study, not considering social-environmental factors in PCC is another gap perceived by subjects participated this 
study. However, various studies in different developed countries have shown that socio-economic status of women plays an important role in maternal and fetal health. Mother's education, family income, and mother's occupational stresses have been related to premature birth and gestational developmental delays $(28,29)$. In their study, Khayatan et al. demonstrated that, from viewpoints of staffs, among factors under study, financial factors with an average of 2.27 had a medium effect on people's access to health services. State and non-state financial support of health services and tariff rates of health services provided in urban health centers have been most effective financial factor (30). In addition, scientists have verified direct effect of work environment on males and females' reproductive system and finally reproductive health (31-33). Therefore, it is important to consider clients' environmental and occupational conditions and give them recommendations on environmental risk factors. Possibly, one of the causes of lack of attention to environmental and occupational risk factors by service providers in preconception counseling sessions is the time allocated to counseling with each client because of shortage of human resources in medical and health centers, in such a way that Jahani Shourab et al. emphasized increasing the number of health providers proportional to the population covered by medical and health centers, along with more notices (34). Therefore, it could be definitely said that the main part of fetal exposure to biological, mental, and environmental risk factors is because of mothers' inappropriate social and economic conditions. Part of this burden can be partly reduced by social support. Participants have had contradictory beliefs on professional ability and skills of PCC providers. Some midwives confessed that they lack sufficient counseling skills. Some women believed midwives working in medical and health centers are of similar or even higher knowledge and skills compared with gynecologists providing PCC. On the other hand, some women regarded knowledge and skills of medical and health centers' staff at a low level, which has resulted in distrust of physicians and midwives working in medical and health centers and services provided by them. In the study conducted by Jahani Shourab et al. in Mashhad Medical and Health Centers, it was shown that providing training in PCC form was not conducted in about half the cases. In addition, assuring patients of confidentially of information, not introducing service providers to a service giver, lack of observing nonverbal skills in creating relations with client were cases less considered when conducting care and counseling. On the other hand, health staff had weak performance on notices and informing clients on the importance of PCC and conducting it in centers (34), which comply with the result of the present study.

\section{Conclusions}

Missing men and adolescents from PCC, insufficient PCC packages, and inadequate PCC strategies and health care providers' incompetency were among the findings of this study. Practical importance of the findings is that filling existing gaps will result in quality improvement in PCC, which finally results in mothers, children, and family health. It is also suggested to provide PCC for adolescents and husbands. Standardization of a care package, especially in terms of counseling for using birth control methods, should be used for clients seeking PCC services. In addition, it is recommended for PCC providers to pay attention to socio-environmental determinants of reproductive and sexual issues in preconception counseling and to earn required skills to conduct counseling by service providers.

\section{Acknowledgments:}

This article is part of a Ph.D. thesis in reproductive health, which has been approved by Mashhad University of Medical Sciences, Mashhad, Iran (with grant no. of 921372). The authors express their gratitude to the vice chancellor for research, Mashhad University of Medical Sciences, Mashhad, Iran.

\section{Conflict of Interest:}

There is no conflict of interest to be declared.

Authors' contributions:

All authors contributed to this project and article equally. All authors read and approved the final manuscript.

\section{References:}

1) Centre BSR. Preconception health: Research and strategies. Toronto, Ontario, Canada; 2001.

2) Program APH. Preconception health framework 2007. Available from: www.aphp.ca/pdf/Preconception Report proof 04.26.07.pdf.

3) Reeve ME. Preconception health: The missing link in the MNCH continuum of care. Beijing Da Xue Xue Bao. 2009; 41(4): 383-8. PMID: 19845066. 
4) Wilkinson H, Trustees and Medical Advisers. Saving mothers' lives. Reviewing maternal deaths to make motherhood safer: 2006-2008. BJOG. 2011; 118(11): 1402-3. doi: 10.1111/j.1471-0528.2011.03097. PMID: 21906238.

5) Mander R, Smith GD. Saving Mothers' Lives (formerly Why Mothers die): Reviewing maternal deaths to make motherhood safer 2003-2005. Midwifery. 2008; 24(1): 8-12. doi: 10.1016/j.midw.2008.01.008. PMID: 18282645.

6) Khosravi A RMR. Indicators of health measures In the Islamic Republic of Iran: Ministry of Health; 2008.

7) Beck S, Wojdyla D, Say L, Betran AP, Merialdi M, Requejo JH, et al. The worldwide incidence of preterm birth: a systematic review of maternal mortality and morbidity. Bulletin of the World Health Organization. 2010; 88(1): 31-8. doi: 10.2471/BLT.08.062554. PMID: 20428351, PMCID: PMC2802437.

8) Korenbrot CC, Steinberg A, Bender C, Newberry S. Preconception care: a systematic review. Matern Child Health J. 2002; 6(2): 75-88. PMID: 12092984.

9) Moosazadeh M, Nekoei moghadam M, Emrani Z, Amiresmaili M. Prevalence of unwanted pregnancy in Iran: a systematic review and meta analysis. Int J Health Plann Manage. 2014; 29(3): 277-90. doi: 10.1002/hpm.2184. PMID: 23630092.

10) Asadi Y, Meshkat M, Talaee B. Determination prevalence and causes of unwanted pregnancy in Mashhad. Med Sci J Islamic Azad University of Mashhad. 2007; 3: 91-5.

11) Mansoory A, Hossainy SH, Dadgar S. Unexpected pregnancy and relative factors in pregnant women referring to Mashhad maternity wards in 2004. Journal of Birjand University of Medical Sciences. 2009; 16(1): 65-70.

12) Posner SF, Broussard DL, Sappenfield WM, Streeter N, Zapata LB, Peck MG. Where are the data to drive policy changes for preconception health and health care? Women's Health Issues. 2008; 18(6): 81-6. doi: 10.1016/j.whi.2008.07.001. PMID: 19059552.

13) Guidelines on reproductive health for the un resident coordinator system United Nations Population $\begin{array}{lllll}\text { Information } & \text { Network } & \text { (POPIN). } & \text { Available } & \text { from: }\end{array}$ http://www.un.org/popin/unfpa/taskforce/guide/iatfreph.gdl.html.

14) Boulet SL, Parker C, Atrash H. Preconception care in international settings. Matern Child Health J 2006; 10(1): 29-35. doi: 10.1007/s10995-006-0091-1. PMID: 16710763, PMCID: PMC1592155.

15) Speziale HS, Streubert HJ, Carpenter DR. Qualitative research in nursing : Advancing the humanistic imperative: Lippincott Williams \& Wilkins; 2011.

16) Burns N, Grove SK. The practice of nursing research: Appraisal, synthesis, and generation of evidence: Saunders Elsevier St Louis^ eMO MO, 2009.

17) Hsieh HF, Shannon SE. Three approaches to qualitative content analysis. Qual Health Res. 2005; 15(9): 1277-88. doi: 10.1177/1049732305276687. PMID: 16204405.

18) Adib Hajbagheri M, Parvizi S, Salsali M. Qualitative research methods. Tehran: Booshra Publication. 2004.

19) Graneheim UH, Lundman B. Qualitative content analysis in nursing research: concepts, procedures and measures to achieve trustworthiness. Nurse Educ Today. 2004; 24(2): 105-12. doi: 10.1016/j.nedt.2003.10.001. PMID: 14769454.

20) Polite DF, Beck CT, Hungler BP. Essentials of nursing research; methods, appraisal and uitilization, 5 ed. Philadelphia: Lippincott Co. 2006.

21) Johnson K, Posner SF, Biermann J, Cordero JF, Atrash HK, Parker CS, et al. Recommendations to improve preconception health and health care- United States. MMWR Recomm Rep. 2006; 55(4): 1-23. PMID: 16617292.

22) Gavin L, Moskosky S, Carter M, Curtis K, Glass E, Godfrey E, et al. Providing quality family planning services: recommendations of CDC and the US Office of Population Affairs. MMWR Recomm Rep. 2014; 63(4): 1-54. PMID: 24759690.

23) Choiriyyah I, Sonenstein FL, Astone NM, Pleck JH, Dariotis JK, Marcell AV. Men Aged 15-44 in Need of Preconception Care. Matern Child Health J. 2015; 19(11): 2358-65. doi: 10.1007/s10995-015-1753-7. PMID: 26112749.

24) Tuomainen H, Cross Bardell L, Bhoday M, Qureshi N, Kai J. Opportunities and challenges for enhancing preconception health in primary care: qualitative study with women from ethnically diverse communities. BMJ open. 2013; 3(7): 2977. doi: 10.1136/bmjopen-2013-002977. PMID: 23883884, PMCID: PMC3731794.

25) Bayrami R, Ebrahimipour H, Ebrahimi M, Froutani MR, Najafzadeh B. Health care provider s' knowledge, attitude and practice regarding pre-conception care. Journal of Research \& Health. 2013; 3(4): 519-26. 
26) Riazi H, Bashirian S, Amini L. Awareness of pregnant women about folic acid supplementation in Iran. Journal of Family and Reproductive Health. 2012; 6(4): 159-63.

27) Merghati Khuiee E, Adab Z, Vahedi F, Farajvand N, Afzalinia T, Rahmanpour L. Compare the Checklist and guidline of preconception care between Iran and some developed countries. Behdad. 2011; 3: 33-5.

28) Teramoto S, Soeda A, Hayashi Y, Urashima M. Physical and socioeconomic predictors of birthweight in Japan. Pediatr Int. 2006; 48(3): 274-7. PMID: 16732794.

29) Cory Slechta DA, Virgolini MB, Thiruchelvam M, Weston DD, Bauter MR. Maternal stress modulates the effects of developmental lead exposure. Environ Health Perspect. 2004; 112(6): 717-30. PMID: 15121516, PMCID: PMC1241967.

30) Khayatan M, Nasiri Pour AA, Amini M, Mohamad Nejad SM. The Effective factors on recievers' access to health care services in urban health care centers. Payavard Salamat. 2011; 4(3): 18-27.

31) Bayrami R, Latifnejad Roudsari R, Mirzaee Rabor F. The principles of preconceptional health promotion. tabriz: elvin. 2012.

32) van den Hazel P, Zuurbier M, Babisch W, Bartonova A, Bistrup ML, Bolte G, et al. Today's epidemics in children: possible relations to environmental pollution and suggested preventive measures. Acta Paediatr Suppl. 2006; 95(453): 18-25. PMID: 17000565.

33) Weselak M, Arbuckle TE, Wigle DT, Walker MC, Krewski D. Pre-and post-conception pesticide exposure and the risk of birth defects in an Ontario farm population. Reprod Toxicol. 2008; 25(4): 472-80. doi: 10.1016/j.reprotox.2008.05.060. PMID: 18586452.

34) Sardasht FG, Shourab NJ , Jafarnejad F, Esmayli H. Comparing the quality of preconception care provided in healthcare centers in Mashhad in 2012. Electron. Physician. 2015; 7(2): 1039-46. doi: 10.14661/2015.1039-1046, PMID: 26120412, PMCID: PMC4477763 